\title{
Mathematical modeling and optimal control of complex epidemiological networks
}

\author{
Guillaume Cantin, Nathalie Verdière
}

\begin{abstract}
An optimal strategy for controlling the dynamics of a multi-scale complex network of epidemiological models is investigated. We focus on the chikungunya epidemic and consider the spreading of the disease within an indigenous urban region contaminated after the transmission of the virus to the local vectorial fauna by a single human individual. Our innovative model takes into account the distinct mobility properties of the vectorial fauna and the human population. The control strategy reproduces the association of a vaccination campaign and a limitation process on the displacements of individuals within the geographical area. Our computations show that the association of both controls is necessary in order to reach an extinction equilibrium of the disease with optimality of the strategy.
\end{abstract}

\section{Introduction}

\subsection{Motivation}

This chapter is devoted to the study of an innovative mathematical model for controlling the dynamics of a complex network of epidemiological models. Our work is motivated by the following observations. First, it is well-known that subtropical diseases can develop new epidemic outbreaks in tempered areas, due to the unintended transportation of a vectorial fauna through aerial or maritime networks. Furthermore, the environmental suitability for the establishment of the disease vectors as the mosquitoes species Aedes Albopictus is promoted by climate changes. All these factors add to the risk for the indigenous transmission of chikungunya in many geographical areas. For example, these factors have played an important role

G. Cantin, N. Verdière Normandie Univ, UNILEHAVRE, LMAH, FR CNRS 3335, ISCN, Le Havre, France e-mail: guillaumecantin@mail.com, nathalie.verdiere@univ-lehavre.fr 
in the chikungunya spread to Brazil in 2013, to South and Central America and the Caribbean islands [25], [16] and to Europe [12], [28]. Furthermore, it has been recently observed that subtropical diseases such as dengue or chikungunya can develop new epidemic outbreaks, in another unexpected way; namely, some individuals can catch the disease during a journey in a subtropical country, and transmit the virus to the local fauna of their native region after their return. Afterwards, the local fauna is susceptible to provoke the spreading of the disease; those new infections are called autochtonous cases. Our aim is to propose a mathematical model which is likely to reproduce a similar situation and to develop an optimal strategy in order to control the spreading of the epidemic. To achieve our goal, we consider an epidemiological model for the chikungunya disease, given by a system of ordinary differential equations (ODE). This system has been studied in [19], [20], [21], [22]; it models the transmission of the chikungunya to human beings by the mosquitoes species Aedes Albopictus; it has been partially calibrated in order to fit with the data of the epidemic on the Réunion island, in the Indian ocean. Considering the complexity of the epidemic, due to the fact that the vectorial fauna and the human population present distinct mobility properties, we construct a multi-scale complex network of non-identical instances of this epidemiological model.

\subsection{Current state of art}

Many papers have been devoted to the analysis of epidemiological models (see for instance [17], [13] or [23] and the references therein). Using agent-based models, microscopic approaches are considered in [18], [26]; those approaches promote various spatial scenarios of vaccination and can give rise to web simulation platforms [29]. In parallel, an increasing number of works study complex networks of epidemiological models (see for instance [21] or [6]). Those models present both a spatial structure and a temporal structure, but they do not always take into account the necessity to be hybrid [3], which shows that the level of refinement of those models can still be improved. They belong to an important class of complex systems models which can be used for modeling a great number of real-world applications, such as neural networks [1], [32] or geographical networks [9], [5], [7]. Emergence and self-organization, such as synchronization, are some of the topics which are usually analyzed [2], [4], [11], [15], [27]. Recently, the problem of controlling the dynamics of this class of models has received an undoubted interest [8]. Nevertheless, many questions are still open in this field. In particular, it is not known if one can control the dynamics of a complex network by a strategy which would focus on the topology of the network. 


\subsection{Organization of the chapter}

Here, we aim to bring a novel contribution to the study of the influence of the topology on the dynamics of complex networks. To this end, we consider a complex network of epidemiological models for the chikungunya and we propose an optimal control strategy for driving the dynamics of the network to an extinction of the disease. In our strategy, we model the smart territories decision making at two different levels. First, we assume that a vaccination campaign is organized; this control seems to be realistic, since recent progress has been realized on finding a vaccine against chikungunya [10]. Vaccination is viewed as an internal command on the dynamics of each place of the geographical area. In parallel, we study the possibility to control human displacements within this area. We focus on a fictional scenario modeling a geographical region with a middle-size city environed by a couple of small cities. We assume that the outbreak takes place in the main city and analyze the spreading of the disease in a large time temporality. However, our framework can be applied to a great number of real-world situations. In the next section, we present the chikungunya transmission model studied in [19] and recall the main properties of its dynamics. In section 3, we show how to construct a multiscale complex network of non-identical instances of this model. In section 4 , we set the optimal control problem with the superposition of vaccination and controls on human displacements. Finally, we present in section 5 numerical computations for simulating the control of an epidemic in a specific geographical territory.

\section{Problem statement}

\subsection{Mathematical modeling of the chikungunya epidemic and transmission model}

The chikungunya disease is an arbovirus mostly transmitted to human beings by mosquitoes species Aedes Albopictus, which was firstly identified in 1953 in eastern Africa. This vector-borne disease admits similarities with the dengue disease and represents a severe public health problem, with an increasing geographical spreading to new countries, due for instance to unintended mobilities of mosquitoes through aerial or maritime networks. In this chapter, we consider an epidemiological model studied in [19], where a growing population of mosquitoes transmits the disease to the human population, which can in turn transmit the disease to uninfected mosquitoes. The mechanisms described by this epidemiological model are depicted in figure 1. The reader who is not familiar with mathematical equations may find sufficient information in this schema. 


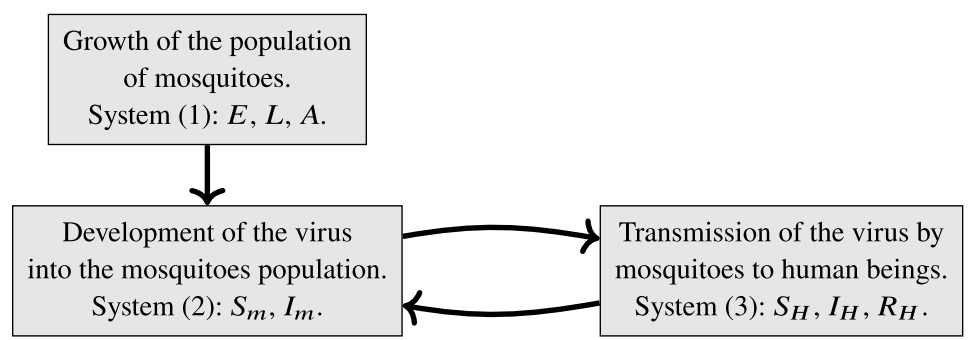

Fig. 1 Transmission of the chikungunya disease from the mosquitoes to human beings.

The model is given by the three following systems of ordinary differential equations:

$$
\begin{aligned}
& \left\{\begin{array}{l}
\frac{d E}{d t}=b A\left(1-\frac{E}{K_{E}}\right)-(s+d) E \\
\frac{d L}{d t}=s E\left(1-\frac{L}{K_{L}}\right)-\left(s_{L}+d_{L}\right) L \\
\frac{d A}{d t}=s_{L} L-d_{m} A
\end{array}\right. \\
& \left\{\begin{array}{l}
\frac{d S_{m}}{d t}=s_{L} L-d_{m} S_{m}-\beta_{m} \frac{I_{H}}{S_{H}+I_{H}+R_{H}} S_{m} \\
\frac{d I_{m}}{d t}=\beta_{m} \frac{I_{H}}{S_{H}+I_{H}+R_{H}} S_{m}-d_{m} I_{m},
\end{array}\right. \\
& \left\{\begin{array}{l}
\frac{d S_{H}}{d t}=-\beta_{H} \frac{I_{m}}{A} S_{H}+b_{H}\left(S_{H}+I_{H}+R_{H}\right)-d_{H} S_{H} \\
\frac{d I_{H}}{d t}=+\beta_{H} \frac{I_{m}}{A} S_{H}-\gamma I_{H}-d_{H} I_{H} \\
\frac{d R_{H}}{d t}=\gamma I_{H}-d_{H} R_{H} .
\end{array}\right.
\end{aligned}
$$

In the latter system (1)-(2)-(3), $E$ denotes the number of eggs in a given population of mosquitoes species Aedes Albopictus at time $t, L$ the number of larva and nymphs and $A$ the number of adult mosquitoes; the three equations of subsystem (1) model the growth of the population of mosquitoes. Next, $S_{m}$ corresponds to the number of adult mosquitoes which are uninfected by the chikungunya virus, but are susceptible to contract it, whereas $I_{m}$ determines the number of infected adult mosquitoes which transmit the virus to the human population. Obviously, we have $A(t)=S_{m}(t)+I_{m}(t)$ for each time $t$. The two equations of subsystem (2) correspond to the development of the virus into the mosquitoes population. Finally, $S_{H}, I_{H}$ and $R_{H}$ correspond to the numbers of uninfected but susceptible human beings, infected human beings and recovered human beings respectively. The three equations of subsystem (3) model the transmission of the virus by mosquitoes to human beings.

The coefficients $b, K_{E}, K_{L}, s, d, s_{l}, d_{L}, d_{m}, b_{H}, \beta_{m}, \beta_{H}, d_{H}$ and $\gamma$ are assumed to be positive; their significance is presented in table 1 . We refer the reader to [19] 
for the detailed presentation of each term of the model. It is proved in [19] that the chikungunya transmission model (1)-(2)-(3) admits relevant solutions, that is, non-negative and bounded solutions whose existence is global.

Table 1 Presentation and significance of the parameters involved in the chikungunya transmission model (1)-(2)-(3).

\begin{tabular}{ll}
\hline Parameter & Significance \\
\hline$b$ & laying rate \\
$K_{E}$ & eggs capacity of the nest \\
$K_{L}$ & larva capacity of the nest \\
$s$ & rate of evolution from egg to larva \\
$s_{L}$ & rate of evolution from larva to mature female \\
$d$ & eggs mortality rate \\
$d_{L}$ & larva mortality rate \\
$d_{m}$ & mature female mortality rate \\
$b_{H}$ & growth rate of human beings \\
$\beta_{m}$ & rate of infection by contact with mosquitoes \\
$\beta_{H}$ & rate of infection by contact with human beings \\
$d_{H}$ & mortality rate of human beings \\
$\gamma$ & rate of immunization \\
\hline
\end{tabular}

\subsection{Extinction or persistence of the disease}

Here, we aim to briefly recall the main ingredients of the dynamics of the transmission model (1)-(2)-(3), before presenting the construction of complex networks of nonidentical instances of that system. Let us introduce the quantity $R_{0}$ given by

$$
R_{0}=\frac{\beta_{m} \beta_{H}}{d_{m}\left(\gamma+b_{H}\right)}
$$

The coefficient $R_{0}$ is usually called basic reproduction number; it corresponds to the average number of individuals contaminated by interaction with a single infected individual. The value of the basic reproduction number $R_{0}$ determines the dynamics of the epidemic. Roughly speaking, if $R_{0}$ admits a low value, then the transmission of the disease into the human population is weak and the disease vanishes. Otherwise, if $R_{0}$ admits a high value, then the disease is likely to spread and persist within the human population. The following theorem describes the dynamics of system (1)-(2)-(3), under the technical assumption

$$
\frac{b s s_{L}}{d_{m}(s+d)\left(s_{L}+d_{L}\right)}>1
$$

which guarantees that the viability of the population of mosquitoes. 
Theorem 0.1 [19] Assume that (5) holds. If $R_{0}<1$, then the transmission model (1)-(2)-(3) admits a unique equilibrium

$$
X_{0}=\left(E^{*}, L^{*}, A^{*}, S_{m}^{*}, I_{m}^{*}, 0,0,0\right) .
$$

If $R_{0}>1$, then the transmission model (1)-(2)-(3) admits a second equilibrium

$$
X^{*}=\left(E^{*}, L^{*}, A^{*}, S_{m}^{*}, I_{m}^{*}, S_{H}^{*}, I_{H}^{*}, R_{H}^{*}\right) .
$$

Furthermore, $X_{0}$ is globally asymptotically stable if and only if $R_{0}<1$ and $X^{*}$ is globally asymptotically stable if $R_{0}>1$. Finally, if $R_{0}>1$ and $b_{H}$ is suffciently small, then the solutions of the transmission model (1)-(2)-(3) oscillate in a neighborhood of $X^{*}$.

The equilibrium $X_{0}$ given by (6) corresponds to the extinction of the disease and exists whatever the values of the parameters are; it is called disease-free equilibrium (DFE). At the opposite, the equilibrium $X^{*}$ given by (7) corresponds to the persistence of the disease within the human population and exists only when the basic reproduction number $R_{0}$ satisfies the condition $R_{0}>1$; it is called endemic equilibrium (EE). Obviously, we aim to control the dynamics of the transmission model (1)-(2)-(3) in order to reach the disease-free equilibrium $X_{0}$. We present in figure 2 two numerical simulations of the transmission model (1)-(2)-(3) which illustrate the alternative described in the latter theorem. The parameters values are given in table 2 and the initial conditions are those of node 1 in table 3.
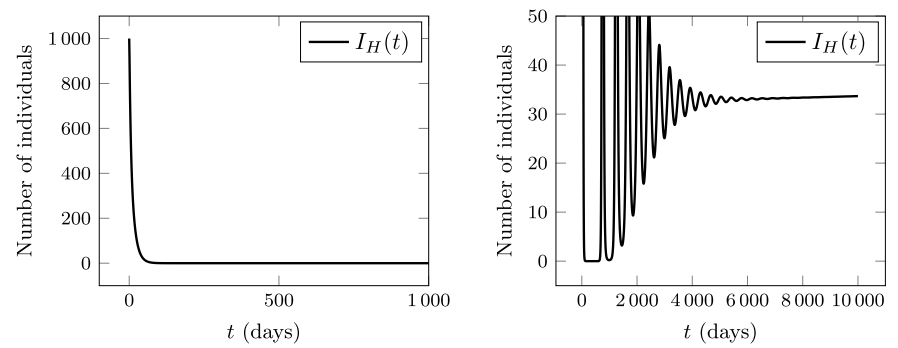

Fig. 2 Dynamics of the transmission model (1)-(2)-(3). Left: if $R_{0}<1$, then the disease-free equilibrium $X_{0}$ is reached by the solutions, so that the number of infected human beings tends to 0 . Right: if $R_{0}>1$ and $b_{H}$ is sufficiently small, then the endemic equilibrium is reached by the solutions after a transitional phase which may exhibit damped oscillations. 


\section{Construction of the complex network problem with a multi-scale topology}

In this section, we show how to construct a complex network with non-identical instances of the transmission model (1)-(2)-(3).

First, we consider a graph $\mathscr{G}$ made with $n$ vertices $(n \geq 2)$ and a given number of edges, which models a geographical area affected by the epidemic. Each vertex models a city, whereas each edge models a connection between two distinct cities. We assume that the populations of mosquitoes and human beings are distributed within those cities. We associate to each vertex of the graph one instance of the transmission model (1)-(2)-(3). To that aim, we introduce the non-linear operators $f_{i}, 1 \leq i \leq n$, defined by

$$
f_{i}\left(x_{i}\right)=\left[\begin{array}{l}
b_{i} A_{i}\left(1-\frac{E_{i}}{K_{E, i}}\right)-\left(s_{i}+d_{i}\right) E_{i} \\
s_{i} E_{i}\left(1-\frac{L_{i}}{K_{L, i}}\right)-\left(s_{L, i}+d_{L, i}\right) L_{i} \\
s_{L, i} L_{i}-d_{m, i} A_{i} \\
s_{L, i} L_{i}-d_{m, i} S_{m, i}-\beta_{m, i} \frac{I_{H, i}}{S_{H, i}+I_{H, i}+R_{H, i}} S_{m, i} \\
\beta_{m, i} \frac{I_{H, i}}{S_{H, i}+I_{H, i}+R_{H, i}} S_{m, i}-d_{m, i} I_{m, i} \\
-\beta_{H, i} \frac{I_{m, i}}{A} S_{H, i}+b_{H, i}\left(S_{H, i}+I_{H, i}+R_{H, i}\right)-d_{H, i} S_{H, i} \\
+\beta_{H, i} \frac{I_{m, i}}{A} S_{H, i}-\gamma_{i} I_{H, i}-d_{H, i} I_{H, i} \\
\gamma_{i} I_{H, i}-d_{H, i} R_{H, i}
\end{array}\right],
$$

where $x_{i}=\left(E_{i}, L_{i}, A_{i}, S_{m, i}, I_{m, i}, S_{H, i}, I_{H, i}, R_{H, i}\right)^{T}$ denotes the state of vertex $i$. In our notation, the subscript $i$ refers to each vertex of the graph $\mathscr{G}$; we assume that two distinct vertices admit distinct parameters, so that they are likely to exhibit distinct dynamics. Indeed, some vertices may admit a low basic reproduction number $\left(R_{0}<1\right)$, whereas some other vertices may admit a high basic reproduction number $\left(R_{0}>1\right)$

Next, our aim is to take into account the distinct mobility properties of mosquitoes and human beings. Thus we distinguish two different scales of mobilities on the same network. To this end, we assume that the set of edges of the graph $\mathscr{G}$ can be split into two subsets $\mathscr{E}_{m}=\left\{e_{m, 1}, \ldots, e_{m, l}\right\}(l \in \mathbb{N})$ and $\mathscr{E}_{H}=\left\{e_{H, 1}, \ldots, e_{H, k}\right\}(k \in \mathbb{N})$, corresponding to the mobilities of mosquitoes and human beings respectively (see figure 3). Additionally, we introduce the matrices of connectivity $m=\left(m_{i, j}\right)_{1 \leq i, j \leq n}$ and $H=\left(H_{i, j}\right)_{1 \leq i, j \leq n}$ by setting 


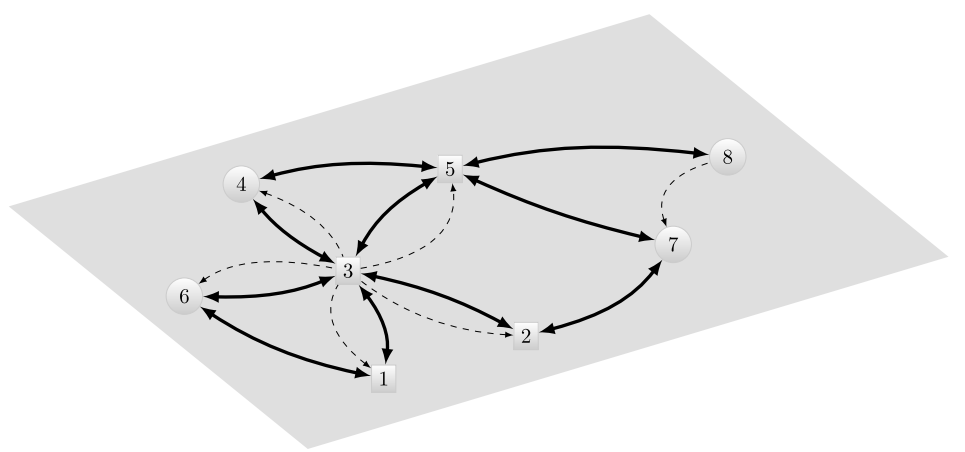

Fig. 3 Complex network modeling the heterogeneous geographical area affected by a given epidemic. The two distinct subsets of edges model distinct mobility properties of disparate populations: dotted edges represent mosquitoes mobility and continuous edges correspond to human beings displacements. Circles correspond to cities such that $R_{0}<1$, whereas squares correspond to cities such that $R_{0}>1$.

$$
\begin{aligned}
& m_{i, j}>0 \text { if }(j, i) \in \mathscr{E}_{m} \text { with } i \neq j, \quad m_{i, j}=0 \text { else, } \quad m_{i, i}=-\sum_{\substack{j=1 \\
j \neq i}}^{n} m_{j, i}, \\
& H_{i, j}>0 \text { if }(j, i) \in \mathscr{E}_{H} \text { with } i \neq j, \quad H_{i, j}=0 \text { else, } \quad H_{i, i}=-\sum_{\substack{j=1 \\
j \neq i}}^{n} H_{j, i}
\end{aligned}
$$

In this way, $m$ and $H$ are matrices of order $n$ whose sum of coefficients of each column is null. We assume that the set of edges $\mathscr{E}_{m} \cup \mathscr{E}_{H}$ does not possess any loop. We also introduce two matrices of coupling strengths $K_{m}$ and $K_{H}$ of orders 5 and 3 respectively, defined by

$$
K_{m}=\operatorname{diag}\left(\varepsilon_{1}, \varepsilon_{2}, \varepsilon_{3}, \varepsilon_{4}, \varepsilon_{5}, 0,0,0\right), \quad K_{H}=\operatorname{diag}\left(0,0,0,0,0, \sigma_{1}, \sigma_{2}, \sigma_{3}\right),
$$

with $\varepsilon_{i} \geq 0(1 \leq i \leq 5)$ and $\sigma_{i} \geq 0(1 \leq i \leq 3)$. We introduce the notations

$$
\begin{aligned}
& K_{m} x_{i}=\left(\varepsilon_{1} E_{i}, \varepsilon_{2} L_{i}, \varepsilon_{3} A_{i}, \varepsilon_{4} S_{m, i}, \varepsilon_{5} I_{m, i}, 0,0,0\right)^{T}, \\
& K_{H} x_{i}=\left(0,0,0,0,0, \sigma_{1} S_{H, i}, \sigma_{2} I_{H, i}, \sigma_{3} R_{H, i}\right)^{T},
\end{aligned}
$$

for $1 \leq i \leq n$. Finally, we define a coupling operator $g=\left(g_{1}, \ldots, g_{n}\right)^{T}$ by setting

$$
g_{i}\left(x_{1}, \ldots, x_{n}\right)=\sum_{j=1}^{n}\left(m_{i, j} K_{m} x_{j}+H_{i, j} K_{H} x_{j}\right), \quad 1 \leq i \leq n .
$$

We emphasize that the coupling operator $g$ has been constructed in order to distinguish and superpose the distinct mobility properties of the mosquitoes and human 
beings, since the transmission model (1)-(2)-(3) involves two populations of individuals of different natures. The coupling strength $\varepsilon_{i}(1 \leq i \leq 5)$ and $\sigma_{i}(1 \leq i \leq 3)$ shall be set to relevant numerical values in section 5 , in order to reproduce the rates of mobilities of mosquitoes and human beings respectively. Typically, the numerical values of $\varepsilon_{i}(1 \leq i \leq 5)$ will be very small with respect to the numerical values of $\sigma_{i}(1 \leq i \leq 3)$, as shown in Table 2. Indeed, human displacements can cover a long distance in a short time, whereas mosquitoes mobility is limited to a few hundred meters in their life (with numerous variations according to the birth environment, see [18]). However, our work intends to take into account the transportation of mosquitoes through human mobility networks, which can increase the distance browsed by mosquitoes [12], [28]. Overall, the rates of displacements represent an average per time of mobility.

With these notations, the complex network of transmission models (1)-(2)-(3) can be written

$$
\frac{d x_{i}}{d t}=f_{i}\left(x_{i}\right)+\sum_{j=1}^{n}\left(m_{i, j} K_{m} x_{j}+H_{i, j} K_{H} x_{j}\right), \quad 1 \leq i \leq n,
$$

or in a short form

$$
\frac{d X}{d t}=F(X)+m K_{m} X+H K_{H} X,
$$

where $X=\left(x_{1}, \ldots, x_{n}\right)^{T}$ and $F(X)=\left(f_{1}\left(x_{1}\right), \ldots, f_{n}\left(x_{n}\right)\right)$.

\section{$>$ Multi-scale mobility}

The transmission model (1)-(2)-(3) involves two disparate populations (mosquitoes and human beings) whose mobility properties are modeled by considering two distinct scales of mobilities on the same network.

Note that in our construction of the complex network problem, we have not normalized the transmission model (1)-(2)-(3), whereas it has been normalized in [19]. The reason is that we aim to construct a complex network modeling an heterogeneous geographical urban area with cities of different sizes.

Using classical methods, we easily establish the existence and uniqueness of solutions for the complex network problem (12). We skip the proof which does not fit with the scope of this textbook.

Theorem 0.2 For any initial condition $X_{0} \in\left(\mathbb{R}^{+}\right)^{8 n}$, the complex network problem (12) admits a unique global solution $X(t)$ stemming from $X_{0}$, whose components are non-negative and bounded for $t \in \mathbb{R}^{+}$. 


\section{Optimal control problem}

In this section, our aim is to propose an optimal control strategy in order to drive the solutions of the complex network problem (12) to an extinction of the disease, while minimizing the cost of the strategy. We assume that the strategy acts on the dynamics of the complex network problem (12) through two leverages, as depicted in figure 4 below.

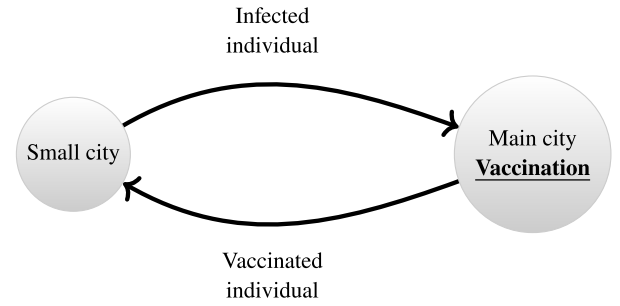

Fig. 4 Example of a strategy to limit the propagation of the virus: association of vaccination in one city and control on the human displacements.

First, we suppose that a vaccination campaign is organized (note that recent progress has been realized on finding a vaccine against chikungunya [10]). This control can be viewed as an internal command of the complex network problem (12), since it involves a control function $u_{0}$ within the operators $f_{i}(1 \leq i \leq n)$, which determine the internal dynamics of each node of the network. For that purpose, we introduce the following notation:

$$
f_{i}\left(x_{i}, u_{0}\right)=f_{i}\left(x_{i}\right)+\left(0,0,0,0,0,0,-u_{0} I_{H, i},+u_{0} I_{H, i}\right)^{T} .
$$

Note that the control function $u_{0}$ appears in the 7-th and 8-th components of $f_{i}\left(x_{i}, u_{0}\right)$. Obviously, many other strategies could be proposed in order to control the internal dynamics of each node of the complex network, such as insecticide treatment for instance (see [20], [24]). Here, we have chosen to focus on a vaccination campaign and to investigate the effect of that campaign, when associated to a control of the displacements of human beings acting in parallel.

\section{$>$ Strategy for controlling the dynamics of the epidemic}

The optimal control strategy of the complex network (12) models the ability of smart territories to act at different levels, since a vaccination campaign and a limitation of human beings displacements involve different civil organizations.

In parallel, we propose a second level of control which acts on the topology of the network, by controlling the displacements of human beings within the geographical 
area. To that aim, we introduce the matrix

$$
H\left(u_{1}, \ldots, u_{k}\right)=\left(H_{i, j}\left(u_{1}, \ldots, u_{k}\right)\right)_{1 \leq i, j \leq n}
$$

defined by

$$
\begin{aligned}
& H_{j, i}\left(u_{1}, \ldots, u_{k}\right)=H_{j, i}+u_{r} \text { if } H_{j, i}>0 \in \mathscr{E}_{H} \text { with } i \neq j, 1 \leq r \leq k \\
& H_{j, i}\left(u_{1}, \ldots, u_{k}\right)=0 \text { else, } \\
& H_{i, i}\left(u_{1}, \ldots, u_{k}\right)=-\sum_{\substack{j=1 \\
j \neq i}}^{n} H_{j, i} .
\end{aligned}
$$

Roughly speaking, the matrix $H\left(u_{1}, \ldots, u_{k}\right)$ is defined by adding a control function $u_{r}$ with $1 \leq r \leq k$ nearby each positive coefficient of matrix $H$ defined by (9) (recall that $k$ denotes the number of edges in $\mathscr{E}_{H}$ ).

With these notations, we consider the general control problem defined by

$$
\frac{d x_{i}}{d t}=f_{i}\left(x_{i}, u_{0}\right)+m K_{m} x_{i}+H\left(u_{1}, \ldots, u_{k}\right) K_{H} x_{i}, \quad 1 \leq i \leq n .
$$

Additionally, we introduce a performance criterion $J$ defined by

$$
J\left(X_{0}, u, T\right)=T+\int_{0}^{T}\left(\sum_{i=1}^{n} I_{H_{i}}^{2}(t)+\sum_{r=0}^{k} u_{r}^{2}(t)\right) d t
$$

where $u=\left(u_{0}, u_{1}, \ldots, u_{k}\right)$. The first term in the performance criterion corresponds to the minimization of time; the second term corresponds to the minimization of infected human beings; the third term corresponds to the minimization of the cost of the control strategy. In this way, we can formulate the optimal control problem: for each initial condition $X_{0} \in\left(\mathbb{R}^{+}\right)^{8 n}$, we search a pair $(X, u)$ defined on an interval $(0, T]$, satisfying the equations (14) for each $t \in(0, T]$, minimizing the performance criterion $J$ defined by (15), so that $X(T)$ belongs to an arbitrary small neighborhood $\mathscr{N}$ of the trivial equilibrium. Applying the general results of existence of optimal control theory (see [14] or [31]) leads to the following theorem. Note that the internal control modeled by a vaccination campaign is necessary: otherwise, the optimal control problem is unfeasible.

Theorem 0.3 For each initial condition $X_{0} \in\left(\mathbb{R}^{+}\right)^{8 n}$ and each arbitrarily small neighborhood $\mathscr{N}$ of the trivial equilibrium, there exists a pair $(X, u)$ defined on an interval $(0, T]$, satisfying the equations (14) for each $t \in(0, T]$, such that $X(T) \in \mathscr{N}$ and minimizing the performance criterion $J$ defined by (15). 


\section{Numerical computations for simulating the control strategy}

In this section, we present numerical simulations for a fictional scenario of an heterogeneous urban area, with a middle-size city environed by three small cities. The corresponding network is depicted in figure 5. We assume that the main city

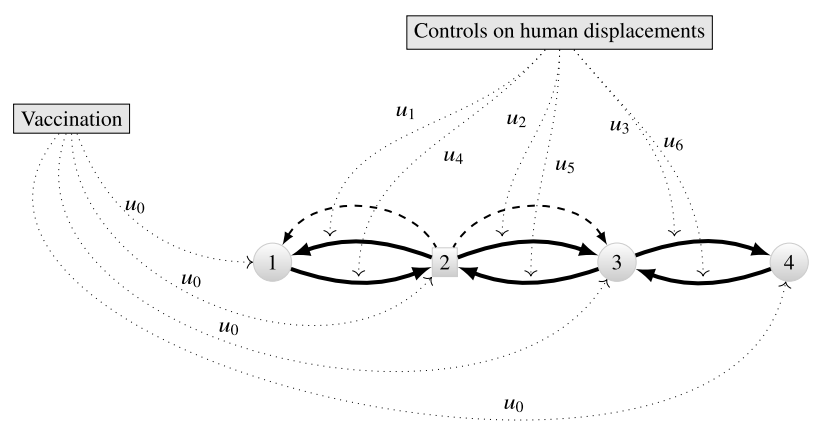

Fig. 5 Schema of a fictional urban area, with a middle-size city (represented by a square) environed by three small cities (represented by circles). Dotted edges correspond to mobility of mosquitoes, whereas continuous edges model human displacements.

admits a high basic reproduction number $R_{0}$, whereas the other cities admit a low basic reproduction number. To this end, we choose different values for the rate $\beta_{H}$ of infection by contact with human beings (see table 2 : $\beta_{H}=5$ in city $2 ; \beta_{H}=0.025$ in cities 1, 3, 4). First, we present the numerical results obtained in absence of control. Then we compare the effect of organizing a vaccination campaign in every city or only in the main city. The values of the parameters and of the initial conditions are given in tables 2 and 3 respectively. Obviously, our framework can be applied to a great number of other scenarios and geographical areas. Computations have been performed with the free and open source software BOCOP [30], on the server of the Laboratory of Applied Mathematics of the University of Le Havre Normandie, in a GNU/Linux environment.

\subsection{Absence of control}

The dynamics of the transmission model (1)-(2)-(3) are predicted by Theorem 0.1 . In the case of the complex network depicted in figure 5 , we observe in absence of control that the nodes of the network admitting a low basic reproduction number, which should converge to the trivial equilibrium, adopt the dynamics of the node which admits a high basic reproduction number (see figure 6). This is characteristic from the synchronization phenomenon of complex network with a symmetric topology 
Table 2 Parameters values for a complex network of transmission models (1)-(2)-(3) (source: [19]).

\begin{tabular}{ll}
\hline Parameter & Value \\
\hline$b$ & 6 \\
$K_{E}$ & 1000 \\
$K_{L}$ & 500 \\
$s$ & 0.7 \\
$s_{L}$ & 0.5 \\
$d, d_{L}$ & 0.2 \\
$d_{m}$ & 0.25 \\
\hline
\end{tabular}

\begin{tabular}{ll}
\hline Parameter & Value \\
\hline$\beta_{m}$ & 0.5 \\
$b_{H}$ & 0.0000457 \\
$\beta_{H}$ & $5\left(R_{0}>1\right)$ or $0.025\left(R_{0}<1\right)$ \\
$d_{H}$ & $0.9 b_{H}$ \\
$\gamma$ & 0.1428 \\
$\varepsilon$ & 0.01 \\
$\sigma$ & 0.1 \\
\hline
\end{tabular}

Table 3 Initial values and end of the transitional dynamics values for a complex network of transmission models (1)-(2)-(3).

\begin{tabular}{cllll}
\hline$t=0$ & Node 1 & Node 2 & Node 3 & Node 4 \\
\hline$E(0)$ & 1 & 1000 & 1 & 1 \\
$L(0)$ & 1 & 1 & 1 & 1 \\
$S_{m}(0)$ & 1 & 1 & 1 & 1 \\
$I_{m}(0)$ & 1 & 1 & 1 & 1 \\
\multicolumn{1}{c}{$S_{H}(0)$} & 10000 & 100000 & 10000 & 10000 \\
$I_{H}(0)$ & 10000 & 10000 & 10000 & 10000 \\
\multicolumn{1}{c}{$R_{H}(0)$} & 1 & 1 & 1 & 1 \\
\hline$t=t_{1}$ & Node 1 & Node 2 & Node 3 & Node 4 \\
\hline$E\left(t_{1}\right)$ & $8.13 \times 10^{2}$ & $7.85 \times 10^{2}$ & $8.13 \times 10^{2}$ & $8.04 \times 10^{2}$ \\
$L\left(t_{1}\right)$ & $3.11 \times 10^{2}$ & $3.02 \times 10^{2}$ & $3.11 \times 10^{2}$ & $3.08 \times 10^{2}$ \\
$S_{m}\left(t_{1}\right)$ & $6.44 \times 10^{2}$ & $5.59 \times 10^{2}$ & $6.45 \times 10^{2}$ & $6.17 \times 10^{2}$ \\
$I_{m}\left(t_{1}\right)$ & $3.79 \times 10^{-1}$ & $6.81 \times 10^{-1}$ & $3.10 \times 10^{-1}$ & $1.12 \times 10^{-1}$ \\
$S_{H}\left(t_{1}\right)$ & $1.28 \times 10^{3}$ & $1.26 \times 10^{3}$ & $1.30 \times 10^{3}$ & $1.32 \times 10^{3}$ \\
$I_{H}\left(t_{1}\right)$ & $1.19 \times 10^{1}$ & $2.86 \times 10^{1}$ & 9.55 & 3.96 \\
$R_{H}\left(t_{1}\right)$ & $4.22 \times 10^{4}$ & $4.22 \times 10^{4}$ & $4.22 \times 10^{4}$ & $4.22 \times 10^{4}$ \\
\hline
\end{tabular}

[2], [4]. This first simulation shows the necessity of a control strategy in order to reach an extinction of the disease in the whole network.

\subsection{Association of vaccination and controls of human displacements}

We continue with the numerical results of the computation of an optimal control $u$ for the complex network problem (12). We assume that the control strategy begins at time $t=t_{1}$, which takes place after the end of the transitional dynamics exhibiting damped oscillations (see figure 6).

First, we assume that a vaccination campaign is organized in every city, which means that the internal control $u_{0}$ is free to be activated in every node of the network. In parallel, the controls on the displacements of human beings within the network are also activated. The computation of an optimal control leads to a value of the 


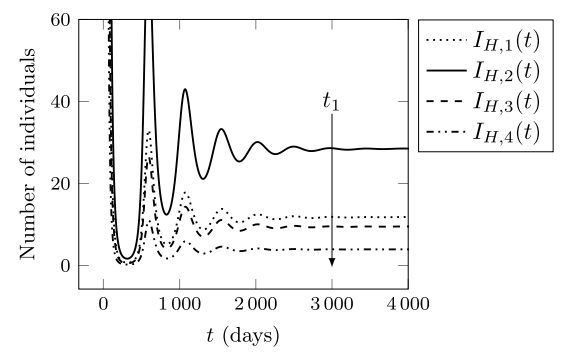

Fig. 6 Numerical results of the complex network depicted in figure 5 in absence of control. The nodes of the network admitting a low basic reproduction number, which should converge to the trivial equilibrium, adopt the dynamics of the node which admits a high basic reproduction number. The transitional dynamics exhibits damped oscillations which vanish after time $t_{1}$.

performance criterion given by $J \simeq 389.444$. The complete results are depicted in figure 7 . We observe that the control strategy is efficient and that the final time of the strategy is very short. Furthermore, the control functions $u_{1}, u_{2}, u_{3}$, which correspond to displacements from center towards periphery, are activated at the beginning of the strategy, whereas the control functions $u_{4}, u_{5}, u_{6}$, which conversely correspond to displacements from periphery to center, are activated at the end of the strategy. Next, we assume that a vaccination campaign is organized in the main city only. The
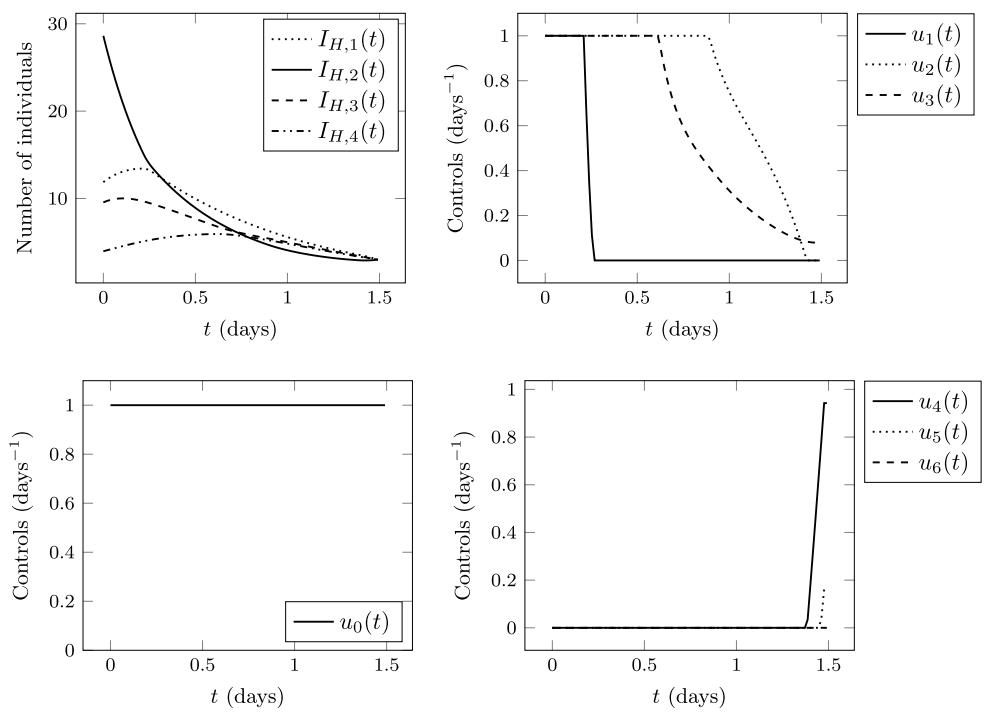

Fig. 7 Numerical results for the optimal control of the complex network depicted in figure 5, when a vaccination campaign is organized in every city. 
computation leads to a value of the performance criterion given by $J \simeq 753.571$, which is approximately the double of the latter performance criterion, as shown in figure 8. Roughly speaking, this value corresponds to the necessity to displace human beings towards the main city in order to be vaccinated, and to come back to the peripheral small cities where the basic reproduction number is more favorable for a decrease of the number of infected human beings. Indeed, it is remarkable to observe that the control function $u_{4}$ has a totally different shape than in the previous case. This might be simply interpreted in the following sense: inhabitants of city (1) have to move towards city (2) in order to receive the vaccination. In parallel, the control function $u_{1}$ remains important, which can correspond for example to a return of individuals who have received the vaccination.
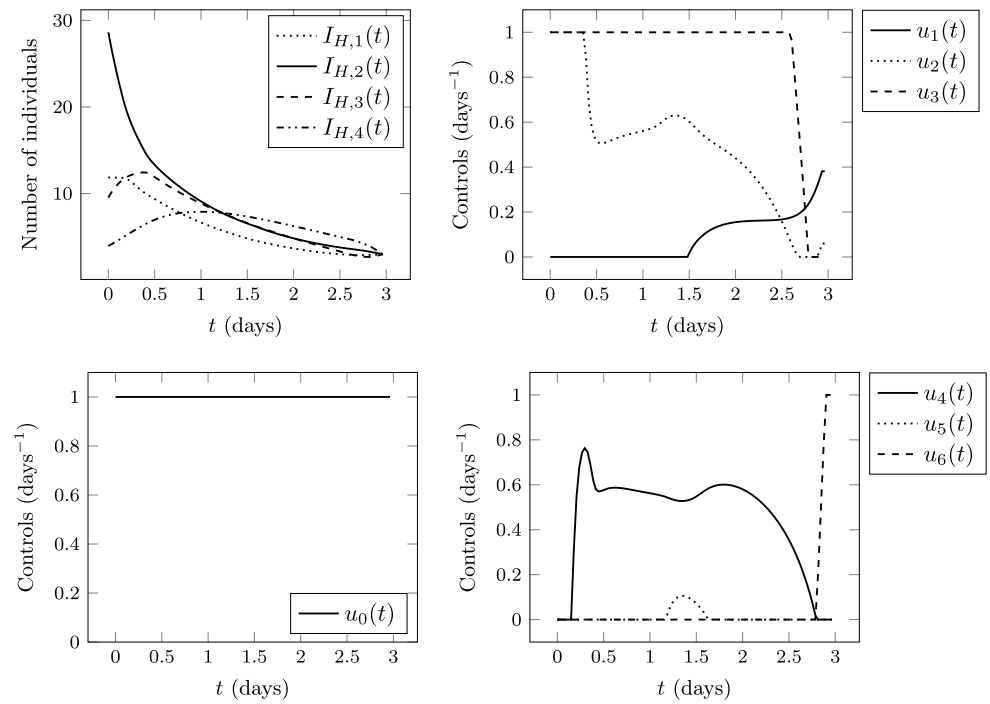

Fig. 8 Numerical results for the optimal control of the complex network depicted in figure 5, when a vaccination campaign is organized only in the main city.

\section{$>$ Feasibility of the strategy}

We emphasize that the internal control modeled by a vaccination campaign [10] appears numerically necessary: otherwise, the optimal control problem is unfeasible. If it is not possible to organize such a vaccination campaign, then the necessity of internal control should be taken into consideration and lead to some other strategy, such as insecticide treatment or awareness campaigns for instance [20], [24]. 
Finally, we emphasize that another control strategy has been studied in [20], where larvicide and insecticide treatments are applied, and prevention campaigns organized in parallel, since there was no vaccine nor specific treatment against chikungunya in 2012. In this paper, the authors mainly conclude: "high application of larvicide or measures to control the proliferation of mosquitoes is needed during all the interval $[0, T]$ even if the peak of epidemic is passed. Then, we observe various scenarios depending on the mosquito population and virus transmission dynamics." The necessity of high application of larvicide in [20] appears similar to the necessity of vaccination in our strategy, at least in one city. Furthermore, the variations of controls depending on the mosquito population observed in [20] are of the same order as the variations of the controls applied to the human displacements. In other words, even if the natures of the strategies are completely different, it appears as a necessity to set an efficient control on the virus itself.

\section{Conclusion}

In this chapter, we have investigated the possibility to control the dynamics of a complex network of epidemiological models for the chikungunya disease. Taking into account the distinct mobilities of human beings and of the vectorial fauna (mosquitoes), we have set a strategy in which we associate vaccination and controls on the human displacements. Our computations, which are validated in a theoretical framework, show that:

- it is not possible to control the propagation of the virus without vaccination;

- it is possible to control the propagation by organizing a vaccination campaign only in the main cities;

- the cost of the control strategy may decrease if vaccination is organized in several cities.

The numerical simulations have been tested on a fictional scenario which is sufficiently general to be applied to a great number of real territories. Furthermore, the methods employed in our work can serve, with appropriate modifications, as a basis of improvement for other epidemiological problems, such as the modeling of the current Covid-19 world pandemic.

In a future work, we aim to improve our approach, by taking into account the variety of behavioral reactions of individuals facing the epidemic; to that aim, we propose to consider a hybrid model [3] with the alliance of microscopic and macroscopic scales.

\section{References}

1. B. Ambrosio, M. Aziz-Alaoui, and V. Phan. Large time behaviour and synchronization of complex networks of reaction-diffusion systems of FitzHugh-Nagumo type. IMA Journal of 
Applied Mathematics, 2019.

2. M. Aziz-Alaoui. Synchronization of chaos. Encyclopedia of Mathematical Physics, Elsevier, 5:213-226, 2006

3. A. Banos, N. Corson, B. Gaudou, V. Laperrière, and S. Coyrehourcq. The importance of being hybrid for spatial epidemic models: a multi-scale approach. Systems, 3(4):309-329, 2015.

4. I. Belykh, M. Hasler, M. Lauret, and H. Nijmeijer. Synchronization and graph topology. International Journal of Bifurcation and Chaos, 15(11):3423-3433, 2005.

5. G. Cantin. Non identical coupled networks with a geographical model for human behaviors during catastrophic events. International Journal of Bifurcation and Chaos, 27(14):1750213, 2017.

6. G. Cantin and C. Joao Silva. Influence of the topology on the dynamics of a complex network of epidemiological systems. AIMS Mathematics Journal, 4:1145, 2019.

7. G. Cantin, N. Verdière, and M. Aziz-Alaoui. Large time dynamics in complex networks of reaction-diffusion systems applied to a panic model. IMA Journal of Applied Mathematics, 09 2019.

8. G. Cantin, N. Verdière, and V. Lanza. Synchronization under control in complex networks for a panic model. In Lecture Notes in Computational Science, ICCS 2019, University of Faro, 2019.

9. G. Cantin, N. Verdière, V. Lanza, M. Aziz-Alaoui, R. Charrier, C. Bertelle, D. Provitolo, and E. Dubos-Paillard. Control of panic behavior in a non identical network coupled with a geographical model. In PhysCon 2017, pages 1-6, University, Firenze, 2017.

10. L.-J. Chang, K. A. Dowd, F. H. Mendoza, J. G. Saunders, S. Sitar, S. H. Plummer, G. Yamshchikov, U. N. Sarwar, Z. Hu, M. E. Enama, et al. Safety and tolerability of chikungunya virus-like particle vaccine in healthy adults: a phase 1 dose-escalation trial. The Lancet, 384(9959):2046-2052, 2014.

11. G. Chen, X. Wang, and X. Li. Fundamentals of complex networks: models, structures and dynamics. John Wiley \& Sons, 2014.

12. E. Delisle, C. Rousseau, B. Broche, I. Leparc-Goffart, G. L'Ambert, A. Cochet, C. Prat, V. Foulongne, J. Ferre, O. Catelinois, et al. Chikungunya outbreak in Montpellier, France, september to october 2014. Eurosurveillance, 20(17):21108, 2015.

13. K. Dietz and K. Hadeler. Epidemiological models for sexually transmitted diseases. Journal of mathematical biology, 26(1):1-25, 1988.

14. W. H. Fleming and R. W. Rishel. Deterministic and stochastic optimal control, volume 1. Springer Science \& Business Media, 2012.

15. M. Golubitsky and I. Stewart. Nonlinear dynamics of networks: the groupoid formalism. Bulletin of the American Mathematical Society, 43(3):305-364, 2006.

16. T. F. Kautz, E. E. Díaz-González, J. H. Erasmus, I. R. Malo-García, R. M. Langsjoen, E. I Patterson, D. I. Auguste, N. L. Forrester, R. M. Sanchez-Casas, M. Hernández-Ávila, et al. Chikungunya virus as cause of febrile illness outbreak, Chiapas, Mexico, 2014. Emerging infectious diseases, 21(11):2070, 2015.

17. W.-M. Liu, H. W. Hethcote, and S. A. Levin. Dynamical behavior of epidemiological models with nonlinear incidence rates. Journal of mathematical biology, 25(4):359-380, 1987.

18. S. Maneerat and E. Daudé. Étude par simulation à base d'agents des effets des discontinuités intra-urbaines à Delhi sur la dispersion des moustiques Aedes aegypti, vecteurs de la dengue, de la fièvre jaune, du chikungunya et du virus Zika. Cybergeo: European Journal of Geography, 2017.

19. D. Moulay. Modélisation et analyse mathématique de systèmes dynamiques en épidémiologie. Application au cas du Chikungunya. PhD thesis, Laboratoire de Mathématiques Appliquées du Havre, 2011.

20. D. Moulay, M. Aziz-Alaoui, and H.-D. Kwon. Optimal control of chikungunya disease: larvae reduction, treatment and prevention. Mathematical Biosciences \& Engineering, 9(2):369-392, 2012.

21. D. Moulay and Y. Pigné. A metapopulation model for chikungunya including populations mobility on a large-scale network. Journal of theoretical biology, 318:129-139, 2013. 
22. D. Moulay, N. Verdière, and L. Denis-Vidal. Identifiablility of parameters in an epidemiologic model modeling the transmission of the chikungunya. 9th International Conference on Modeling, Optimization \& SIMulation, Bordeaux, France, 2012.

23. J. Murray. Mathematical Biology I: An Introduction, vol. 17 of Interdisciplinary Applied Mathematics. Springer, New York, NY, USA, 2002.

24. M. Nelder, B. Kesavaraju, A. Farajollahi, S. Healy, I. Unlu, T. Crepeau, A. Ragavendran, D. Fonseca, and R. Gaugler. Suppressing aedes albopictus, an emerging vector of dengue and chikungunya viruses, by a novel combination of a monomolecular film and an insect-growth regulator. The American journal of tropical medicine and hygiene, 82(5):831-837, 2010.

25. R. Omarjee, C. Prat, O. Flusin, S. Boucau, B. Tenebray, O. Merle, P. Huc-Anais, S. Cassadou, and I. Leparc-Goffart. Importance of case definition to monitor ongoing outbreak of chikungunya virus on a background of actively circulating dengue virus, St Martin, December 2013 to January 2014. Eurosurveillance, 19(13):20753, 2014.

26. D. Philippon, M. Choisy, A. Drogoul, B. Gaudou, N. Marilleau, P. Taillandier, and Q. C. Truong. Exploring trade and health policies influence on Dengue spread with an Agent-Based Model. In International Workshop on Multi-Agent Systems and Agent-Based Simulation, pages 111-127. Springer, 2016.

27. B. Rink and J. Sanders. Coupled cell networks: semigroups, Lie algebras and normal forms. Transactions of the American Mathematical Society, 367(5):3509-3548, 2015.

28. T. Seyler, C. Rizzo, A. Finarelli, C. Po, P. Alessio, V. Sambri, M. C. Degli Atti, and S. Salmaso. Autochthonous chikungunya virus transmission may have occurred in Bologna, Italy, during the summer 2007 outbreak. Eurosurveillance, 13(3):3-4, 2008.

29. P. Taillandier, B. Gaudou, A. Grignard, Q.-N. Huynh, N. Marilleau, P. Caillou, D. Philippon, and A. Drogoul. Building, composing and experimenting complex spatial models with the gama platform. GeoInformatica, 23(2):299-322, 2019.

30. I. S. Team Commands. Bocop: an open source toolbox for optimal control. http://bocop.org, 2017.

31. E. Trélat. Contrôle optimal: théorie \& applications, volume 865. Vuibert Paris, France, 2008.

32. X. Yang, J. Cao, and Z. Yang. Synchronization of coupled reaction-diffusion neural networks with time-varying delays via pinning-impulsive controller. SIAM Journal on Control and Optimization, 51(5):3486-3510, 2013. 